\title{
Extending the parking space
}

\author{
Andrew Berget $\|^{1}$ and Brendon Rhoades $\|^{2}$ \\ ${ }^{1}$ Dept. of Mathematics, University of Washington, Seattle, WA, USA \\ ${ }^{2}$ Dept. of Mathematics, University of California - San Diego, La Jolla, CA, USA
}

\begin{abstract}
The action of the symmetric group $S_{n}$ on the set $\operatorname{Park}_{n}$ of parking functions of size $n$ has received a great deal of attention in algebraic combinatorics. We prove that the action of $S_{n}$ on Park P $_{n}$ extends to an action of $S_{n+1}$. More precisely, we construct a graded $S_{n+1}$-module $V_{n}$ such that the restriction of $V_{n}$ to $S_{n}$ is isomorphic to Park ${ }_{n}$. We describe the $S_{n}$-Frobenius characters of the module $V_{n}$ in all degrees and describe the $S_{n+1}$-Frobenius characters of $V_{n}$ in extreme degrees. We give a bivariate generalization $V_{n}^{(\ell, m)}$ of our module $V_{n}$ whose representation theory is governed by a bivariate generalization of Dyck paths. A Fuss generalization of our results is a special case of this bivariate generalization.

Résumé. L'action du groupe symétrique $S_{n}$ sur l'ensemble $\operatorname{Park}_{n}$ des fonctions de stationnement de longueur $n$ a reçu beaucoup d'attention dans la combinatoire algébrique. Nous démontrons que l'action de $S_{n} \operatorname{sur}^{\text {Park }_{n}} \mathrm{~s}^{\text {'étend }}$ à une action de $S_{n+1}$. Plus précisément, nous construisons un gradué $S_{n+1}$-module $V_{n}$ telles que la restriction de $S_{n}$ est isomorphe à $\operatorname{Park}_{n}$. Nous décrivons la $S_{n}$-Frobenius caractères des modules $V_{n}$ à tous les degrés et décrivent le $S_{n+1}$-Frobenius caractères de $V_{n}$ en degrés extrêmes. Nous donnons une généralisation bivariée $V_{n}^{(\ell, m)}$ de notre module $V_{n}$ dont la représentation théorie est régi par une généralisation bivariée des chemins de Dyck. Une généralisation Fuss de nos résultats est un cas particulier de cette généralisation bivariée.
\end{abstract}

Keywords: parking functions, symmetric group, Dyck paths, representation, matriod

\section{Introduction}

This paper is about extending the visible permutation action of $S_{n}$ on the space Park $_{n}$ spanned by parking functions of size $n$ to a hidden action of the larger symmetric group $S_{n+1}$. The $S_{n+1}$-module we construct will be a subspace of the coordinate ring of the reflection representation of type $\mathrm{A}_{n}$ and will inherit the polynomial grading of this coordinate ring. Using statistics on Dyck paths, we will give an explicit combinatorial formula for the graded $S_{n}$-Frobenius character of our module and will describe the extended $S_{n+1}$-Frobenius character in extreme degrees.

As far as the authors know, this is the first example and proof of an extension of Park $_{n}$ to $S_{n+1}$ an $S_{n+1}$-module.

We remark that our result is the 'best possible' in two senses. First, it is not always possible to extend Park $_{n}$ to an $S_{n+2}$-module; for example, the action of $S_{4}$ on Park 4 does not extend to an action of $S_{6}$.

\footnotetext{
${ }^{\dagger}$ Email: abergetemath. washington.edu

$\ddagger$ Email: bprhoades@math.ucsd.edu

1365-8050 @ 2013 Discrete Mathematics and Theoretical Computer Science (DMTCS), Nancy, France
} 
Also, from a combinatorial point of view, one may be interested in extending the action of $S_{n}$ on Park ${ }_{n}$ to a permutation action of the larger symmetric group $S_{n+1}$. Our extended module is graded, but is not a permutation module. However, it is impossible to extend the action of $S_{4}$ on Park 4 to a permutation action of $S_{5}$.

\section{Background and Main Results}

A length $n$ sequence $\left(a_{1}, \ldots, a_{n}\right)$ of positive integers is called a parking function of size $n$ if its nondecreasing rearrangement $\left(b_{1} \leq \cdots \leq b_{n}\right)$ satisfies $b_{i} \leq i$ for all $i$. Parking functions were introduced by Konheim and Weiss [KW66] in the context of computer science, but have seen much application in algebraic combinatorics with connections to Catalan combinatorics, Shi hyperplane arrangements, diagonal coinvariant rings, and rational Cherednik algebras. The set of parking functions of size $n$ is famously counted by $(n+1)^{n-1}$. The $\mathbb{C}$-vector space Park ${ }_{n}$ spanned by the set of parking functions of size $n$ carries a natural permutation action of the symmetric group $S_{n}$ on $n$ letters:

$$
w \cdot\left(a_{1}, \ldots, a_{n}\right)=\left(a_{w(1)}, \ldots, a_{w(n)}\right)
$$

for $w \in S_{n}$ and $\left(a_{1}, \ldots, a_{n}\right) \in \operatorname{Park}_{n}$.

A partition $\lambda$ of a positive integer $n$ is a weakly decreasing sequence $\lambda=\left(\lambda_{1} \geq \cdots \geq \lambda_{k}\right)$ of nonnegative integers which sum to $n$. We write $\lambda \vdash n$ to mean that $\lambda$ is a partition of $n$ and define $|\lambda|:=n$. We call $k$ the length of the partition $\lambda$. The Ferrers diagram of $\lambda$ consists of $\lambda_{i}$ left justified boxes in the $i^{t h}$ row from the top ('English notation'). If $\lambda$ is a partition, we define a new partition mult $(\lambda)$ whose parts are obtained by listing the (positive) part multiplicities in $\lambda$ in weakly decreasing order. For example, we have that mult $(4,4,3,3,3,1,0,0)=(3,2,2,1)$.

We will make use of two partial orders on partitions in this paper. The first partial order is Young's lattice with relations given by $\lambda \subseteq \mu$ if $\lambda_{i} \leq \mu_{i}$ for all $i \geq 1$ (where we append an infinite string of zeros to the ends of $\lambda$ and $\mu$ so that these inequalities make sense). Equivalently, we have that $\lambda \subseteq \mu$ if and only if the Ferrers diagram of $\lambda$ fits inside the Ferrers diagram of $\mu$. Dominance order on partitions is defined by $\lambda \preceq \mu$ if for all $i \geq 1$ we have the inequality of partial sums $\lambda_{1}+\cdots+\lambda_{i} \leq \mu_{1}+\cdots+\mu_{i}$ (where we again append an infinite string of zeros to the ends of $\lambda$ and $\mu$ ). Observe that either of the relations $\lambda \subseteq \mu$ or $\lambda \preceq \mu$ imply that $|\lambda| \leq|\mu|$.

For a partition $\lambda=\left(\lambda_{1}, \ldots, \lambda_{k}\right) \vdash n$, we let $S_{\lambda}$ denote the Young subgroup $S_{\lambda_{1}} \times \cdots \times S_{\lambda_{k}}$ of $S_{n}$. We denote by $M^{\lambda}$ the coset representation of $S_{n}$ given by $M^{\lambda}:=\operatorname{Ind}_{S_{\lambda}}^{S_{n}}\left(\mathbf{1}_{S_{\lambda}}\right) \cong{ }_{S_{n}} \mathbb{C} S_{n} / S_{\lambda}$ and we denote by $S^{\lambda}$ the irreducible representation of $S_{n}$ labeled by the partition $\lambda$.

Let $R_{n}$ denote the $\mathbb{C}$-vector space of class functions $S_{n} \rightarrow \mathbb{C}$. Identifying modules with their characters, the set $\left\{S^{\lambda}: \lambda \vdash n\right\}$ forms a basis of $R_{n}$. The graded vector space $R:=\bigoplus_{n>0} R_{n}$ attains the structure of a $\mathbb{C}$-algebra via the induction product $S^{\lambda} \circ S^{\mu}:=\operatorname{Ind}_{S_{n} \times S_{m}}^{S_{n+m}}\left(S^{\lambda} \otimes_{\mathbb{C}} S^{\mu}\right)$, where $\lambda \vdash n$ and $\mu \vdash m$.

We denote by $\Lambda$ the ring of symmetric functions (in an infinite set of variables $X_{1}, X_{2}, \ldots$, with coefficients in $\mathbb{C}$ ). The $\mathbb{C}$-algebra $\Lambda$ is graded and we denote by $\Lambda_{n}$ the homogeneous piece of degree $n$. Given a partition $\lambda$, we denote the corresponding Schur function by $s_{\lambda}$ and the corresponding complete homogeneous symmetric function by $h_{\lambda}$.

The Frobenius character is the graded $\mathbb{C}$-algebra isomorphism Frob : $R \rightarrow \Lambda$ induced by setting $\operatorname{Frob}\left(S^{\lambda}\right)=s_{\lambda}$. It is well known that we have $\operatorname{Frob}\left(M^{\lambda}\right)=h_{\lambda}$. Generalizing slightly, if $V=$ $\bigoplus_{k \geq 0} V(k)$ is a graded $S_{n}$-module, define $\operatorname{grFrob}(V ; q) \in \Lambda \otimes_{\mathbb{C}} \mathbb{C}[[q]]$ to be the formal power series in $q$ with coefficients in $\Lambda$ given by $\operatorname{grFrob}(V ; q):=\sum_{k \geq 0} \operatorname{Frob}(V(k)) q^{k}$. 


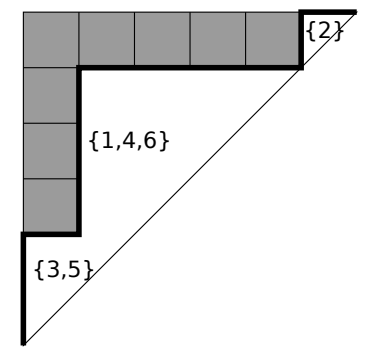

Fig. 1: A Dyck path of size 6 .

A Dyck path of size $n$ is a lattice path $D$ in $\mathbb{Z}^{2}$ consisting of vertical steps $(0,1)$ and horizontal steps $(1,0)$ which starts at $(0,0)$, ends at $(n, n)$, and stays weakly above the line $y=x$. A maximal continguous sequence of vertical steps in $D$ is called a vertical run of $D$.

We will associate two partitions to a Dyck path $D$ of size $n$. The vertical run partition $\lambda(D) \vdash n$ is obtained by listing the (positive) lengths of the vertical runs of $D$ in weakly decreasing order. For example, if $D$ is the Dyck path in Figure 1 , then $\lambda(D)=(3,2,1)$. The area partition $\mu(D)$ is the partition of length $n$ whose Ferrers diagram is the set of boxes to the upper left of $D$ in the $n \times n$ square with lower left coordinate at the origin. For example, if $D$ is the Dyck path of size 6 in Figure 1 , then $\mu(D)=(5,1,1,1,0,0)$. The boxes in the Ferrers diagram of $\mu(D)$ are shaded. We define the area statistic(i) on Dyck paths by area $(D)=|\mu(D)|$. For the Dyck path in our running exampe, area $(D)=8$. By construction, we have that mult $(\mu(D))=\lambda(D)$ for any Dyck path $D$ of size $n$.

Dyck paths of size $n$ can be used to obtain a decomposition of Park $_{n}$ as a direct sum of coset modules $M^{\lambda}$. In particular, let $D$ be a Dyck path of size $n$. A labeling of $D$ assigns each vertical run of $D$ to a subset of $[n]:=\{1,2, \ldots, n\}$ of size equal to the length of that vertical run such that every letter in $[n]$ appears exactly once as a label of a vertical run. Figure 1 shows an example of a labeled Dyck path of size 6 , where the subsets labeling the vertical runs are placed just to the right of the runs.

The set of labeled Dyck paths of size $n$ carries an action of $S_{n}$ given by label permutation. There is an $S_{n}$-equivariant bijection from the set of labeled Dyck paths $D$ of size $n$ to parking functions $\left(a_{1}, \ldots, a_{n}\right)$ of size $n$ given by letting $a_{i}$ be one greater than the $x$-coordinate of the vertical run of $D$ labeled by $i$. For example, the labeled Dyck path in Figure 1 corresponds to the parking function $(2,6,1,2,1,2) \in$ Park $_{6}$. Since any fixed labeled Dyck path of size $D$ generates a cyclic $S_{n}$-module isomorphic to $M^{\lambda(D)}$, it is immediate that the parking space $\operatorname{Park}_{n}$ decomposes into coset representations as

$$
\operatorname{Park}_{n} \cong S_{n} \bigoplus_{D} M^{\lambda(D)}
$$

where the direct sum is over all Dyck paths $D$ of size $n$. Equivalently, we have that the Frobenius character of $\operatorname{Park}_{n}$ is given by Frob $\left(\operatorname{Park}_{n}\right)=\sum_{D} h_{\lambda(D)}$. For example, the 5 Dyck paths of size 3 shown in Figure 3 lead to the Frobenius character

$$
\operatorname{Frob}\left(\operatorname{Park}_{3}\right)=h_{(3)}+3 h_{(2,1)}+h_{(1,1,1)} .
$$

\footnotetext{
(i) Many authors instead define the area of a Dyck path $D$ to be the number of complete lattice squares between $D$ and the line $y=x$, so that our statistic would be the 'coarea'.
} 


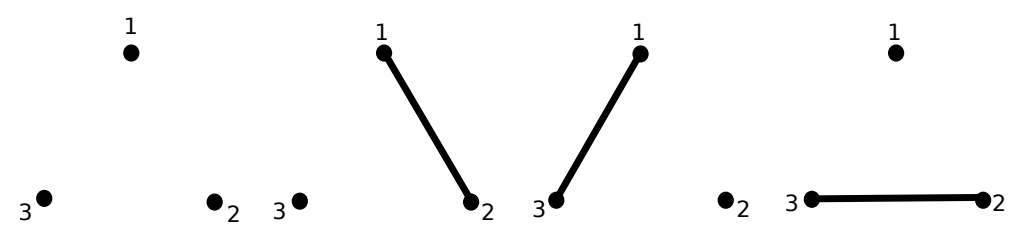

Fig. 2: The four slim subgraphs of $K_{3}$.

The vector space underlying the $S_{n+1}$-module which will extend Park ${ }_{n}$ is a subspace of the polynomial ring $\mathbb{C}\left[x_{1}, \ldots, x_{n+1}\right]$ in $n+1$ variables and first studied in the work of Postnikov and Shapiro [PS04]. Let $K_{n+1}$ denote the complete graph on the vertex set $[n+1]$. Given an edge $e=(i<j)$ in $K_{n+1}$, we associate the polynomial weight $p(e):=x_{i}-x_{j} \in \mathbb{C}\left[x_{1}, \ldots, x_{n+1}\right]$. A subgraph $G \subseteq K_{n+1}$ (identified with its edge set) gives rise to the polynomial weight $p(G):=\prod_{e \in G} p(e)$. Following Postnikov and Shapiro, we call a subgraph $G \subseteq K_{n+1}$ slim if the complement edge set $K_{n+1}-G$ is a connected graph on the vertex set $[n+1]$.

Definition 1 Denote by $V_{n}$ the $\mathbb{C}$-linear subspace of $\mathbb{C}\left[x_{1}, \ldots, x_{n+1}\right]$ given by

$$
V_{n}:=\operatorname{span}\left\{p(G): \text { is a slim subgraph of } K_{n+1}\right\} .
$$

Let $V_{n}(k)$ denote the homogeneous piece of $V_{n}$ of polynomial degree $k$; the space $V_{n}(k)$ is spanned by those polynomials $p(G)$ corresponding to slim subgraphs $G$ of $K_{n+1}$ with $k$ edges.

In the case $n=2$, Figure 2 shows that four slim subgraphs of the complete graph $K_{3}$. From left to right, the corresponding polynomials are $1, x_{1}-x_{2}, x_{1}-x_{3}$, and $x_{2}-x_{3}$. It follows that $V_{2}(0)=\operatorname{span}\{1\}$ and $V_{2}(1)=\operatorname{span}\left\{x_{1}-x_{2}, x_{1}-x_{3}, x_{2}-x_{3}\right\}$. Observe that the graded Frobenius character of $V_{2}$ is $\operatorname{grFrob}\left(V_{2} ; q\right)=s_{(3)} q^{0}+s_{(2,1)} q^{1}$. By the branching rule for symmetric groups (see [Sag01]), we have that $\operatorname{grFrob}\left(\operatorname{Res}_{S_{2}}^{S_{3}}\left(V_{2}\right) ; q\right)=s_{(2)} q^{0}+\left(s_{(2)}+s_{(1,1)}\right) q^{1}$. Setting $q=1$ yields $\operatorname{Frob}\left(\operatorname{Res}_{S_{2}}^{S_{3}}\left(V_{2}\right)\right)=$ $2 s_{(2)}+s_{(1,1)}$, which agrees with the Frobenius character of Park .

While the set of polynomials $\left\{p(G): G\right.$ is a slim subgraph of $\left.K_{n+1}\right\}$ is linearly dependent in general, a basis for $V_{n}$ can be constructed using standard matroid theoretic results. Fix a total order on the edge set of $K_{n+1}$. Given a spanning tree $T$ of $K_{n+1}$, the external activity $\operatorname{ex}(T)$ of $T$ is the set of edges $e \in K_{n+1}$ such that $e$ is the minimal edge of the unique cycle in $T \cup\{e\}$. A basis of $V_{n}$ is given by $\left\{p\left(K_{n+1}-(T \cup \operatorname{ex}(T))\right): T\right.$ is a spanning tree of $\left.K_{n+1}\right\}$. It follows immediately from Cayley's theorem that $\operatorname{dim} V_{n}=(n+1)^{n-1}$.

Since the slimness of a subgraph is preserved under the action of $S_{n+1}$ on the vertex set $[n+1]$ and $p(G)$ is homogeneous of degree equal to the number of edges in $G$, it follows that $V_{n}=\bigoplus_{k>0} V_{n}(k)$ is a graded $S_{n+1}$-submodule of the polynomial ring $\mathbb{C}\left[x_{1}, \ldots, x_{n+1}\right]$. In fact, the space $V_{n}$ sits inside the copy of the coordinate ring of the reflection representation of type $\mathrm{A}_{n}$ sitting inside $\mathbb{C}\left[x_{1}, \ldots, x_{n+1}\right]$ generated by $x_{i}-x_{i+1}$ for $1 \leq i \leq n$.

The following result was conjectured by the first author. We postpone its proof, along with the proofs of the other results in this section, to Section 3

Theorem 2 Embed $S_{n}$ into $S_{n+1}$ by letting $S_{n}$ act on the first $n$ letters. We have that

$$
\operatorname{Res}_{S_{n}}^{S_{n+1}}\left(V_{n}(k)\right) \cong{ }_{S_{n}} \bigoplus_{D} M^{\lambda(D)},
$$



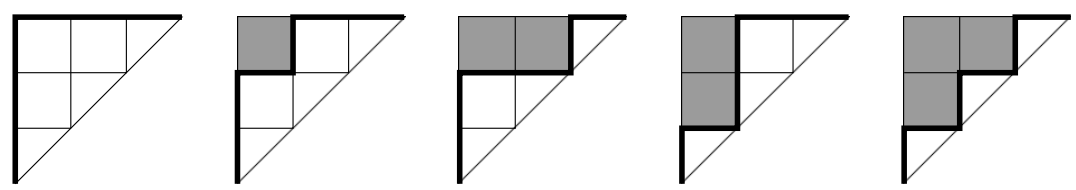

Fig. 3: The 5 Dyck paths of size 3. From left to right, their contributions to the graded Frobenius character $\operatorname{grFrob}\left(\operatorname{Res}_{S_{3}}^{S_{4}}\left(V_{3}\right) ; q\right)$ are $h_{(3)} q^{0}, h_{(2,1)} q^{1}, h_{(2,1)} q^{2}, h_{(2,1)} q^{2}$, and $h_{(1,1,1)} q^{3}$.

where the direct sum is over all Dyck paths of size $n$ and area $k$. In particular, by Equation 2 we have that

$$
\operatorname{Res}_{S_{n}}^{S_{n+1}}\left(V_{n}\right) \cong S_{n} \operatorname{Park}_{n}
$$

Equivalently, we have that $\operatorname{grFrob}\left(\operatorname{Res}_{S_{n}}^{S_{n+1}}\left(V_{n}\right) ; q\right)=\sum_{D} q^{\text {area }(D)} h_{\lambda(D)}$, where the sum is over all Dyck paths $D$ of size $n$. For example, computing the area and run partitions of the 5 Dyck paths of size 3 shown in Figure 3 shows that

$$
\operatorname{grFrob}\left(\operatorname{Res}_{S_{3}}^{S_{4}}\left(V_{3}\right) ; q\right)=h_{(3)} q^{0}+h_{(2,1)} q^{1}+2 h_{(2,1)} q^{2}+h_{(1,1,1)} q^{3} .
$$

Postnikov and Shapiro showed that the dimension of the vector space $V_{n}$ is equal to $(n+1)^{n-1}$, however the $S_{n}$-module structure of $V_{n}$ has remained unstudied. Indeed, Theorem 2 is the first description of the $S_{n}$-module structure of $V_{n}$.

It is natural to ask for an explicit description of the $S_{n+1}$-structure of $V_{n}$ or of its graded pieces $V_{n}(k)$. This problem is open in general, but we can describe the extended structure of $V_{n}(k)$ in the extreme degrees $k=0,1, \ldots, n$ as well as $k=\left(\begin{array}{l}n \\ 2\end{array}\right)$. Let $C_{n+1}$ be the cyclic subgroup of $S_{n+1}$ generated by the long cycle $c:=(1,2, \ldots, n+1)$ and let $\zeta$ be the linear representation of $C_{n+1}$ which sends $c$ to $e^{\frac{2 \pi i}{n+1}}$. Mackey's Theorem can be used to prove that the Lie representation $\operatorname{Lie}_{n}:=\operatorname{Ind}_{C_{n+1}}^{S_{n+1}}(\zeta)$ of $S_{n+1}$ satisfies $\operatorname{Res}_{S_{n}}^{S_{n+1}}\left(\operatorname{Lie}_{n}\right) \cong S_{n} \mathbb{C}\left[S_{n}\right]$. Stanley proved that the Lie representation arises as the action of $S_{n+1}$ on the top poset cohomology of the lattice of set partitions of $[n+1]$, tensored with the sign representation [Sta82].

Proposition 3 The module $V_{n}(0)$ carries the trivial representation of $S_{n+1}$, the module $V_{n}(1)$ carries the reflection representation of $S_{n+1}$, and in general $V_{n}(k)=\operatorname{Sym}^{k}\left(V_{n}(1)\right)$ for $k<n$. The module $V_{n}\left(\left(\begin{array}{l}n \\ 2\end{array}\right)\right)=V_{n}($ top $)$ carries the Lie representation of $S_{n+1}$ tensor the sign representation.

The first part of this result is optimal in the sense that if $k \geq n$ then $V_{n}(k)$ is a proper subspace of $\operatorname{Sym}^{k}\left(V_{n}(1)\right)$.

We will prove a bivariate generalization of Theorem 2 which includes a 'Fuss generalization' as a special case. Given $\ell, m, n>0$, define a $(\ell, m)$-Dyck path of size $n$ to be a lattice path $D$ in $\mathbb{Z}^{2}$ consisting of vertical steps $(0,1)$ and horizontal steps $(1,0)$ which starts at $(-\ell+1,0)$, ends at $(m n, n)$, and stays weakly above the line $y=\frac{x}{m}$. Taking $\ell=m=1$, we recover the classical notion of a Dyck path of size $n$. Taking $\ell=1$ and $m$ general, the $(1, m)$-Dyck paths are the natural Fuss extension of Dyck paths. As before, we define the vertical run partition $\lambda(D) \vdash n$ of an $(\ell, m)$-Dyck path $D$ of size $n$ to be the partition obtained by listing the lengths of the vertical runs of $D$ in weakly decreasing order. We also define the area partition $\mu(D)$ to be the length $n$ partition whose Ferrers diagram fits between $D$ and a 


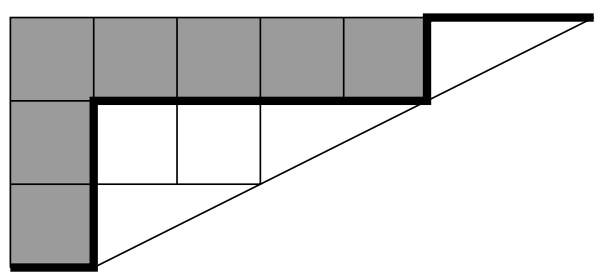

Fig. 4: A $(2,2)$-Dyck path of size 3 .

$(\ell-1+m n) \times n)$ rectangle with lower left hand coordinate $(-\ell+1,0)$. The area of $D$ is defined by area $(D):=|\mu(D)|$. We have that $\operatorname{mult}(\mu(D))=\lambda(D)$.

Figure 4 shows an example of a $(2,2)$-Dyck path of size 3 . The path $D$ starts at $(-1,0)$, ends at $(6,3)$, and stays above the line $y=\frac{x}{2}$. We have that $\lambda(D)=(2,1) \vdash 3, \mu(D)=(5,1,1)$, and area $(D)=7$.

Let $K_{n+1}^{(\ell, m)}$ be the multigraph on the vertex set $[n+1]$ with $m$ edges between $i$ and $j$ for all $1 \leq i<$ $j \leq n$ and $\ell$ edges between $i$ and $n+1$ for all $1 \leq i \leq n$. We call a sub-multigraph $G$ of $K_{n+1}^{(\ell, m)}$ slim if the multi-edge set difference $K_{n+1}^{(\ell, m)}-G$ is a connected multigraph on $[n+1]$. We extend the polynomial weight $p(G) \in \mathbb{C}\left[x_{1}, \ldots, x_{n+1}\right]$ to multigraphs $G$ in the obvious way.

Definition 4 Let $V_{n}^{(\ell, m)}$ be the $\mathbb{C}$-linear subspace of $\mathbb{C}\left[x_{1}, \ldots, x_{n+1}\right]$ given by the span

$$
V_{n}^{(\ell, m)}:=\operatorname{span}\left\{p(G): G \text { is a slim sub-multigraph of } K_{n+1}^{(\ell, m)}\right\}
$$

As in the case $m=\ell=1$, the space $V_{n}^{(\ell, m)}$ is stable under the action of $S_{n}$ and is a graded $S_{n^{-}}$ representation with respect to the standard polynomial degree. When $\ell=m, V_{n}^{(\ell, m)}$ also caries an action of $S_{n+1}$. Postnikov and Shapiro showed that the dimension of $V_{n}^{(\ell, m)}$ is $(m n+\ell)^{n-1}[$ PS04]. Let $V_{n}^{(\ell, m)}(k)$ be the degree $k$ piece of $V_{n}^{(\ell, m)}$.

Theorem 5 We have that

$$
\left(V_{n}^{(\ell, m)}(k)\right) \cong S_{n} \bigoplus_{D} M^{\lambda(D)},
$$

where the direct sum is over all $(\ell, m)$-Dyck paths of size $n$ and area $k$. For $k<n, V_{n}^{(\ell, m)}(k)=$ $\operatorname{Sym}^{k}\left(V_{n}^{(\ell, m)}(1)\right)$.

When $\ell=m$, the top degree piece of $V_{n}^{(\ell, m)}$ is isomorphic, as an $S_{n+1}$-module, to $\operatorname{Lie}_{n} \otimes \operatorname{sign}^{\otimes \ell}$.

While the degree 0 and 1 pieces of $V_{n}^{(\ell, m)}$ have $S_{n+1}$-structure given by the trivial representation and the reflection representation, respectively, the authors do not know of a nice expression for the extended Frobenius character in other degrees.

\section{Proofs}

While Theorem 5 implies Theorem 2, the proof of Theorem 5 is a straightforward extension of the proof of Theorem 2 and it will be instructive to prove Theorem 2 first. 

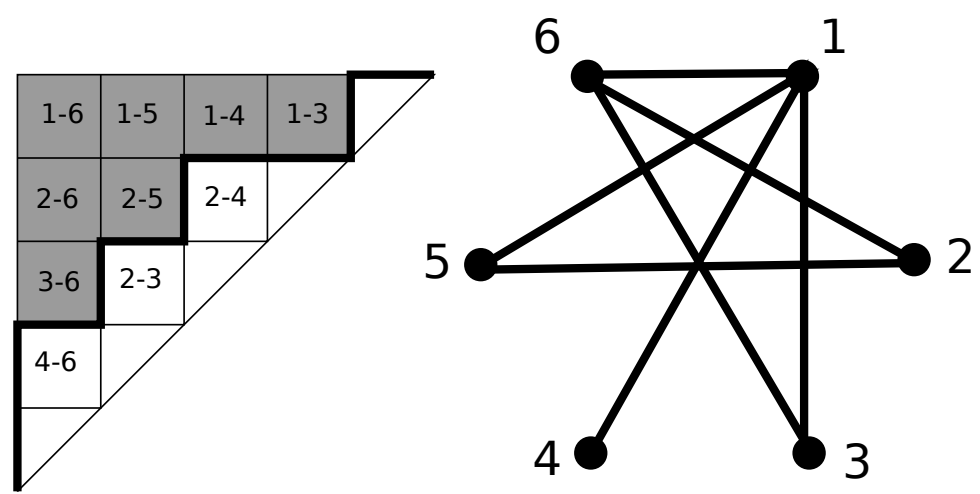

Fig. 5: A Dyck path $D$ of size 5 and the associated subgraph $G(D)$ of $K_{6}$.

The first step in the proof of Theorem 2 is to relate the modules on both sides of the claimed isomorphism by associating a subgraph $G(D)$ of $K_{n+1}$ and a polynomial $p(D) \in \mathbb{C}\left[x_{1}, \ldots, x_{n+1}\right]$ to any Dyck path $D$ of size $n$. We start by labeling the $1 \times 1$ box $b$ which is completely above the line $y=x$ with the edge $e(b)=(n-j, n-i)$ in $K_{n+1}$, where $(i, j)$ is the upper left coordinate of $b$. See Figure 5 for an example of this labeling in the case $n=5$. We let $G(D)$ be the subgraph of $K_{n+1}$ consisting of those edges $e(b)$ for which the box $b$ is to the upper left of the path $D$. In Figure 5. the shaded boxes above the path $D$ each contribute an edge to the subgraph $G(D)$ and we have that $G(D)=\{1-6,1-5,1-4,1-3,2-6,2-5,3-6\}$.

Lemma 6 The subgraph $G(D)$ is slim for any Dyck path $D$.

Proof: The subgraph $G(D)$ contains none of the edges in the path $1-2-\cdots-(n+1)$.

By Lemma 6 the polynomial $p(D):=p(G(D))$ is contained in $V_{n}$. For example, if $n=5$ and $D$ is the Dyck path shown in Figure 5, we have that

$$
p(D)=\left(x_{1}-x_{6}\right)\left(x_{1}-x_{5}\right)\left(x_{1}-x_{4}\right)\left(x_{1}-x_{3}\right)\left(x_{2}-x_{6}\right)\left(x_{2}-x_{5}\right)\left(x_{3}-x_{6}\right) \in V_{5} .
$$

By construction, for any Dyck path $D$ the polynomial $p(D)$ is homogeneous with degree equal to area $(D)$.

In order to prove the direct sum decomposition in Theorem 2, we will show that the polynomials $p(D)$ project nicely onto a certain subspace of $\mathbb{C}\left[x_{1}, \ldots, x_{n+1}\right]$. Since Theorem 2 only concerns the restriction of $V_{n}$ to $S_{n}$, it is natural to consider a subspace of $\mathbb{C}\left[x_{1}, \ldots, x_{n+1}\right]$ which is closed under the action of $S_{n}$ but not of $S_{n+1}$.

Let $s t_{n}:=(n-1, n-2, \ldots, 1)$ be the staircase partition of length $n-1$. We call a partition $\lambda=\left(\lambda_{1}, \ldots, \lambda_{n}\right)$ sub-staircase if $\lambda \subseteq s t_{n}$ (observe that this definition has tacit dependence on $n$ ). For any Dyck path $D$ of size $n$, the partition $\mu(D)$ is sub-staircase.

For a partition $\lambda=\left(\lambda_{1}, \ldots, \lambda_{n}\right)$, we use the shorthand $x^{\lambda}:=x_{1}^{\lambda_{1}} \cdots x_{n}^{\lambda_{n}} \in \mathbb{C}\left[x_{1}, \ldots, x_{n}\right]$. We call a monomial $x_{1}^{d_{1}} \cdots x_{n+1}^{d_{n+1}}$ in the variables $x_{1}, \ldots, x_{n+1}$ sub-staircase if there exists a permutation $w \in S_{n}$ and a sub-staircase partition $\lambda=\left(\lambda_{1} \geq \cdots \geq \lambda_{n}\right) \vdash n$

$$
x_{1}^{d_{1}} \cdots x_{n+1}^{d_{n+1}}=w \cdot x^{\lambda} .
$$


In particular, the variable $x_{n+1}$ does not appear in any sub-staircase monomial. If the monomial $x_{1}^{d_{1}} \cdots x_{n+1}^{d_{n+1}}$ is sub-staircase, the partition $\lambda$ is uniquely determined from the monomial; call this the exponent partition of the monomial. Let $W_{n} \subset \mathbb{C}\left[x_{1}, \ldots, x_{n+1}\right]$ be the $\mathbb{C}$-linear span of all sub-staircase monomials. The subspace $W_{n}$ is closed under the action of $S_{n}$, but not under the action of $S_{n+1}$.

In the case $n=3$, the $S_{3}$-orbits of the 16 staircase monomials in $\mathbb{C}\left[x_{1}, \ldots, x_{4}\right]$ are shown in the following table, where the left column shows a representative from each orbit.

\begin{tabular}{|l||l|}
\hline 1 & \\
$x_{1}$ & $x_{2}, x_{3}$ \\
$x_{1}^{2}$ & $x_{2}^{2}, x_{3}^{2}$ \\
$x_{1} x_{2}$ & $x_{1} x_{3}, x_{2} x_{3}$ \\
$x_{1}^{2} x_{2}$ & $x_{1}^{2} x_{3}, x_{2}^{2} x_{1}, x_{2}^{2} x_{3}, x_{3}^{2} x_{1}, x_{3}^{2} x_{2}$ \\
\hline
\end{tabular}

The $S_{3}$-orbits are parametrized by sub-staircase partitions $\lambda=\left(\lambda_{1}, \lambda_{2}, \lambda_{3}\right)$ and each orbit contains a unique representative of the form $x^{\lambda}$. The staircase monomials form a linear basis of $W_{3}$ and the cyclic $S_{3}$-submodule of $W_{3}$ generated by $x^{\lambda}$ is isomorphic to $M^{\text {mult }(\lambda)}$. The natural bijection between exponent vectors and parking functions affords an isomorphism $W_{3} \cong_{S_{3}}$ Park $_{3}$. These observations generalize in a straightforward way to the following lemma, whose proof is left to the reader.

Lemma 7 The set of sub-staircase monomials forms a linear basis for $W_{n}$ and is closed under the action of $S_{n}$. The $S_{n}$-orbits are parametrized by sub-staircase partitions $\lambda$, and the orbit labeled by $\lambda$ has a unique monomial of the form $x^{\lambda}$. The cyclic $S_{n}$-submodule of $W_{n}$ generated by $x^{\lambda}$ is isomorphic to $M^{\text {mult( }(\lambda)}$ and we have that $W_{n} \cong{ }_{S_{n}} \operatorname{Park}_{n}$.

With Lemma 7 in mind, we will construct a graded $S_{n}$-module isomorphism $V_{n} \stackrel{\sim}{\rightarrow} W_{n}$. We define a graded $S_{n}$-module homomorphism $\phi: V_{n} \rightarrow W_{n}$ by the following composition:

$$
\phi: V_{n} \hookrightarrow \mathbb{C}\left[x_{1}, \ldots, x_{n+1}\right] \rightarrow \mathbb{C}\left[x_{1}, \ldots, x_{n}\right] \rightarrow W_{n},
$$

where the first map is inclusion, the second is the specialization $x_{n+1}=0$, and the third linear map fixes the space $W_{n}$ pointwise and sends monomials which are not sub-staircase to zero.

We want to show that $\phi$ is an isomorphism. Postnikov and Shapiro showed that $\operatorname{dim}\left(W_{n}\right)=\operatorname{dim}\left(V_{n}\right)=$ $(n+1)^{n-1}$ [PS04], so it is enough to show that $\phi$ is surjective. We will do this by analyzing the polynomials $\phi(p(D))$, where $D$ is a Dyck path of size $n$.

The set of sub-staircase partitions forms an order ideal in dominance order. The next lemma states that the transition matrix between the set $\{\phi(p(D)): D$ a Dyck path of size $n\}$ expands in the monomial basis of $W_{n}$ given by $\left\{x^{\lambda}: \lambda\right.$ sub-staircase $\}$ in a unitriangular way with respect to any linear extension of dominance order (where we associate $\phi(p(D)$ ) with the partition $\mu(D)$ ).

Lemma 8 Let $D$ be a Dyck path of size $n$. There exist integers $c_{\lambda, w} \in \mathbb{Z}$ such that

$$
\phi(p(D))=x^{\mu(D)}+\sum_{\substack{\lambda \prec \mu(D) \\|\lambda|=|\mu(D)| \\ w \in S_{n}}} c_{\lambda, w} w \cdot x^{\lambda} .
$$


Proof: By definition, we have that

$$
p(D)=\prod_{e=(i<j) \in G(D)}\left(x_{i}-x_{j}\right),
$$

so (up to sign) a typical monomial in the expansion of $p(D)$ is obtained by choosing an endpoint of every edge in $G(D)$ and multiplying the corresponding variables. The map $\phi$ kills any monomial which contains the variable $x_{n+1}$, so up to sign a typical monomial in $\phi(p(D))$ is obtained by choosing an endpoint of each edge in $G(D)$ and multiplying the corresponding variables such that whenever $G(D)$ has an edge of the form $(i<n+1)$, we choose the smaller endpoint $i$. The result follows from the construction of $G(D)$ and the definition of dominance order.

As an example of Lemma 8, consider the case $n=5$ and let the Dyck path $D$ be shown in Figure 5 with $\mu(D)=(4,2,1)$. To calculate $\phi(p(D))$, we set $x_{6}=0$ in the product formula for $p(D)$ given in Equation 10 and expand. The resulting polynomial is

$$
\begin{aligned}
\phi(p(D)) & =x_{1}\left(x_{1}-x_{5}\right)\left(x_{1}-x_{4}\right)\left(x_{1}-x_{3}\right) x_{2}\left(x_{2}-x_{5}\right) x_{3} \\
& =x_{1}^{4} x_{2}^{2} x_{3}+\text { terms involving sub-staircase monomials with exponent partition } \prec(4,2,1) .
\end{aligned}
$$

We are ready to complete the proof of Theorem 2 .

Proof of Theorem 2; By Lemma 7, the set of sub-staircase monomials forms a linear basis of $W_{n}$, so Lemma 8 implies that the $S_{n}$-module homomorphism $\phi: V_{n} \rightarrow W_{n}$ is surjective. Since $\operatorname{dim}\left(V_{n}\right)=$ $\operatorname{dim}\left(W_{n}\right)$, this implies that $\phi$ is also injective and gives an isomorphism $\operatorname{Res}_{S_{n}}^{S_{n+1}}\left(V_{n}\right) \cong{ }_{S_{n}} \operatorname{Park}_{n}$. To prove the graded isomorphism in Theorem 2, it is enough to observe that $\operatorname{mult}(\mu(D))=\lambda(D)$ for any Dyck path $D$ and apply Lemmas 7 and 8 together with the fact that $\phi$ is graded.

It may be tempting to guess that $p(D)$ generates a cyclic $S_{n}$-submodule of $V_{n}$ isomorphic to $M^{\lambda(D)}$, but this is false in general. The reason for this is that while the 'leading term' in the expansion of $\phi(p(D))$ in Lemma 8 generates the submodule $M^{\lambda(D)}$ under the action of $S_{n}$, the other terms in this expansion can cause $\phi(p(D))$ to generate a different cyclic submodule.

We are ready to prove the claimed $S_{n+1}$-structure of the extreme degrees of the graded module $V_{n}(k)$.

Proof of Proposition 3: It is clear from the definitions that $V_{n}(0)$ carries the trivial representation of $S_{n+1}$. The space $V_{n}(1)$ has basis given by the polynomials $x_{1}-x_{2}, x_{2}-x_{3}, \ldots, x_{n}-x_{n+1}$ and hence carries the reflection representation of $S_{n+1}$ (i.e., the irreducible $S_{n+1}$-module corresponding to the partition $(n, 1)$ ). Since $V_{n} \subseteq \operatorname{Sym}\left(V_{n}(1)\right)$ we are claiming that in degree $k<n$ this is an equality. The Hilbert series of $V_{n}$ is the Tutte polynomial evaluation $q^{\left(\begin{array}{c}n+1 \\ 2\end{array}\right)-n} T_{K_{n+1}}(1,1 / q)$ and so we must prove that the first $n-1$ terms of this sum are the binomial coefficents $\left(\begin{array}{c}n+k-1 \\ k\end{array}\right)$. There is nothing special about $K_{n+1}$ in this claim and we will prove a more general statement in Lemma99

To prove that $V_{n}$ (top) is isomorphic to $\mathrm{Lie}_{n+1} \otimes \operatorname{sign}$ we reason as follows. The space $V_{n}$ (top) is spanned by those $p(G)$ where the complementary subgraph $K_{n+1} \backslash G$ is connected and has $n$ edges.

Let $\mathcal{A}_{n}$ denote the braid arrangementin $\mathbb{C}^{n+1}$, which is the union of those hyperplanes with at least two coordinates equal. Let $H^{*}\left(\mathbb{C}^{n+1} \backslash \mathcal{A}_{n} ; \mathbb{C}\right)$ denote the (complexified) de Rham cohomology ring of its 
complement. Consider, now, the linear map $c: V_{n}($ top $) \rightarrow H^{n}\left(\mathbb{C}^{n+1} \backslash \mathcal{A}_{n}\right)$ that sends

$$
p(G) \mapsto p(G) \cdot d\left(x_{1}-x_{2}\right) \wedge d\left(x_{2}-x_{3}\right) \wedge \cdots \wedge d\left(x_{n}-x_{n+1}\right) / \prod_{1 \leq i<j \leq n}\left(x_{i}-x_{j}\right) .
$$

This is an isomorphism of vector spaces, since it is division by the Vandermond product, followed by multiplication by the $n$-form. To see that $c$ is equivariant notice that $\bigwedge^{n} V_{n}(1)$ carries the sign representation of $S_{n+1}$, because it is 1 dimensional and non-trivial. Likewise does the one dimensional representation spanned by the Vadnermond product. It follows that the signs introduced by multiplication by the $n$-form and division by the Vandermond cancel, and $c$ is equivariant.

Finally, the top degree cohomology of the complement $\mathbb{C}^{n+1} \backslash \mathcal{A}_{n}$ is known to be $S_{n+1}$-isomorphic to the top degreee Whitney homology of its lattice of flats. The lattice of flats of $\mathcal{A}_{n}$ is the partition lattice $\Pi_{n+1}$ and by a result of Stanley [Sta82] (beautifuly recounted by Wachs in [Wac07]), the top degree Whitney homology of the partition lattice $\Pi_{n+1}$ is $\mathrm{Lie}_{n+1} \otimes$ sign.

Lemma 9 Let $G$ be a connected graph on $v$ vertices with e edges. Denote the Tutte polynomial of $G$ by $T_{G}(x, y)$. Then, the polynomial $q^{e-v+1} T_{G}(1,1 / q)$ takes the form,

$$
1+(v-1) q+\left(\begin{array}{l}
v \\
2
\end{array}\right) q^{2}+\left(\begin{array}{c}
v+1 \\
3
\end{array}\right) q^{3}+\cdots+\left(\begin{array}{c}
(v-1)+(v-2)-1 \\
v-2
\end{array}\right) q^{v-2}+O\left(q^{v-1}\right) .
$$

Proof: We write $T_{G}(x, y)$ in terms of the two variable coboundary polynomial, $\bar{\chi}_{G}(\lambda, \nu)$. This is the sum

$$
\bar{\chi}_{G}(\lambda, \nu)=\frac{1}{\lambda} \sum_{i=0}^{e} c_{i}(G ; \lambda) \nu^{i}
$$

where $c_{i}(G ; \lambda)$ is the number of ways to color the vertices of $G$ with $\lambda$ colors and exactly $i$ monochromatic edges. It is a fact that this is a polynomial in $\lambda$ and $\nu$. Now by [Whi92, Theorem 6.3.26],

$$
q^{e-v+1} T_{G}(1,1 / q)=\frac{q^{e}}{(1-q)^{v-1}} \bar{\chi}_{G}(0,1 / q) .
$$

Thus, to prove the first part of the lemma we will show that $c_{i}(G ; \lambda)=0$ for $e-v+1<i<e$, and that $c_{e}(G ; \lambda)=\lambda$. Suppose that we have colored the vertices of $G$ and we have more than $e-v+1$ monochromatic edges. Then the collection of monochromatic edges forms a connected subgraph of $G$. It follows that all vertices of $G$ are colored the same and hence all edges of $G$ are monochromatic. This means that $c_{i}(G ; \lambda)=0$ unless $i=e$. That $c_{e}(G ; \lambda)=\lambda$ is clear.

The proof of Theorem 5 is a straight-forward extension of the proof of Theorem 2 and is only sketched.

Proof of Theorem 5, sketch: Given any $(\ell, m)$-Dyck path $D$ of size $n$ we associate a sub-multigraph $G(D)$ of $K_{n+1}^{(\ell, m)}$ by letting every box which contributes to area $(D)$ correspond to a single edge in the multigraph $G(D)$; the labeling which accomplishes this is shown in Figure 6 in the case $(\ell, m)=(3,2)$ and $n=4$. For general $\ell, m$, and $n$, we label the boxes in the $i^{t h}$ row from the top from left to right with $(\ell+m-2)$ copies of the edge $i-(n+1), m$ copies of the edge $i-n, m$ copies of the edge $i-(n-1), \ldots$, $m$ copies of the edge $i-(i+2)$, and $(m-1)$ copies of the edge $i-(i+1)$. 


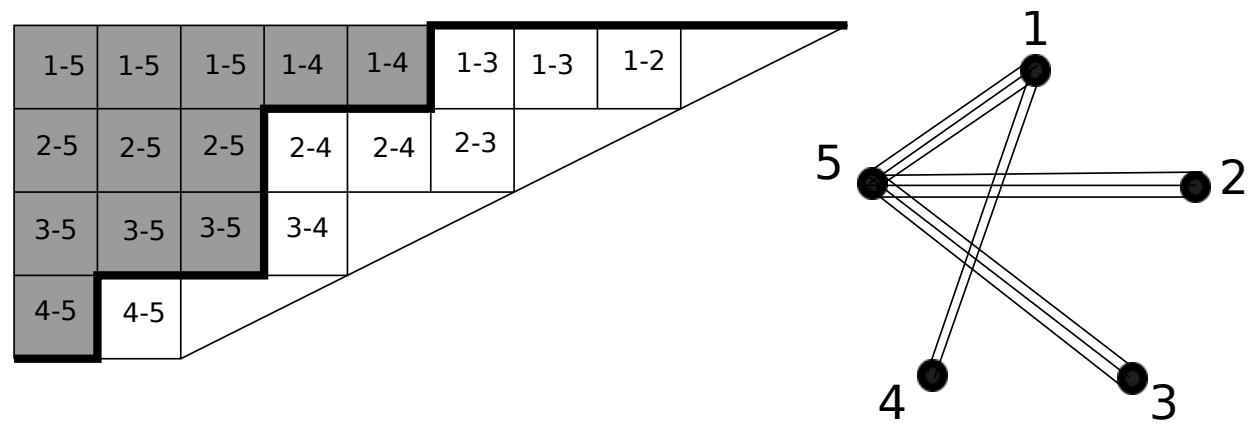

Fig. 6: A $(3,2)$-Dyck path $D$ of size 4 and the associated sub-multigraph $G(D)$ of $K_{4}^{(3,2)}$.

For any $(\ell, m)$-Dyck path $D$ of size $n$, the multigraph complement of $G(D)$ within $K_{n}^{(\ell, m)}$ contains each of the edges in the path $1-2-\cdots-n-(n+1)$ with multiplicity at least one. Therefore, the sub-multigraph $G(D)$ is slim and the polynomial $p(D):=p(G(D))$ is contained in $V_{n}^{(\ell, m)}$.

We say that a partition $\lambda$ with $n$ parts is sub-( $\ell, m)$-staircase if in Young's lattice we have the relation $\lambda \subseteq(\ell-1+m(n-1), \ell-1+m(n-2), \ldots, \ell-1)$. A monomial $x_{1}^{d_{1}} \cdots x_{n+1}^{d_{n+1}}$ is $s u b-(\ell, m)$-staircase if there exists $w \in S_{n}$ and a sub- $(\ell, m)$-staircase partition $\lambda$ such that

$$
x_{1}^{d_{1}} \cdots x_{n+1}^{d_{n+1}}=x_{w(1)}^{\lambda_{1}} \cdots x_{w(n)}^{\lambda_{n}} .
$$

Let $W_{n}^{(\ell, m)}$ be the subspace of $\mathbb{C}\left[x_{1}, \ldots, x_{n+1}\right]$ spanned by the set of all sub- $(\ell, m)$-staircase monomials. We have that $W_{n}^{(\ell, m)}$ is closed under the action of $S_{n}$ and the degree $k$ homogeneous piece of $W_{n}^{(\ell, m)}$ is isomorphic as an $S_{n}$-module to the direct sum on the right hand side of the isomorphism asserted in Theorem 5

The isomorphism in Theorem 5 is proven by showing that the graded $S_{n}$-module homomorphism $\phi^{(\ell, m)}: V_{n}^{(\ell, m)} \rightarrow W_{n}^{(\ell, m)}$ given by the composite

$$
\phi^{(\ell, m)}: V_{n}^{(\ell, m)} \hookrightarrow \mathbb{C}\left[x_{1}, \ldots, x_{n+1}\right] \rightarrow \mathbb{C}\left[x_{1}, \ldots, x_{n}\right] \rightarrow W_{n}^{(\ell, m)}
$$

is an isomorphism, where the first map is inclusion, the second is the evaluation $x_{n+1}=0$, and the third fixes $W_{n}^{(\ell, m)}$ pointwise and sends every monomial which is not sub- $(\ell, m)$-staircase to zero.

Postnikov and Shapiro proved that the vector space $V_{n}^{(\ell, m)}$ has dimension $(\ell+m n)^{n-1}$ [PS04]. A standard counting argument shows that there are $(\ell+m n)^{n-1}$ sub- $(\ell, m)$-staircase monomials, so we have that $\operatorname{dim}\left(V_{n}^{(\ell, m)}\right)=\operatorname{dim}\left(W_{n}^{(\ell, m)}\right)$. Therefore, to show that $\phi^{(\ell, m)}$ is a graded isomorphism of $S_{n}$-modules, it is enough to show that $\phi^{(\ell, m)}$ is surjective.

To show that $\phi^{(\ell, m)}$ is surjective, we prove a generalization of Lemma 8 which states that for any $(\ell, m)$-Dyck path $D$ of size $n$, the monomial expansion of $\phi^{(\ell, m)}(p(D))$ has the form

$$
\phi^{(\ell, m)}(p(D))=x^{\mu(D)}+\text { terms involving monomials whose exponent partitions are } \prec \mu(D),
$$

where we extend the definition of $\mu(D)$ to $(\ell, m)$-Dyck paths of size $n$ in the obvious way. This triangularity result implies that $\phi^{(\ell, m)}$ is surjective, and dimension counting implies that $\phi^{(\ell, m)}$ is a graded $S_{n}$-module isomorphism. Theorem 5 follows. 
The claim about $V_{n}^{(\ell, m)}$ in low degree follows since $V_{n} \subset V_{n}^{(\ell, m)}$. The claim about $V_{n}^{(\ell, \ell)}$ (top) follows since this space is isomorphic to $V_{n}$ (top), the isomorphism being division by $\prod_{i<j}\left(x_{i}-x_{j}\right)^{\ell-1}$.

\section{Concluding Remarks}

In this paper we constructed a graded $S_{n+1}$-module $V_{n}$ which satisfies $\operatorname{Res}_{S_{n}} S_{n+1}\left(V_{n}\right) \cong{ }_{S_{n}} \operatorname{Park}_{n}$. As we mentioned in Section 11 there does not exist an $S_{6}$-module $M$ such that $\operatorname{Res}_{S_{4}}^{S_{6}}(M) \cong_{S_{4}} \operatorname{Park}_{4}$, so we cannot hope for an extension of $\mathrm{Park}_{n}$ to a symmetric group of higher rank than $n+1$ in general.

On the other hand, we identified the top degree $V_{n}$ (top) of $V_{n}$ with the Lie representation $\operatorname{Lie}_{n}$ of $S_{n+1}$ tensor the sign representation. Whitehouse [Whi97] proved that the representation $\mathrm{Lie}_{n}$ extends to $S_{n+2}$. This suggests the following problem.

Problem 10 For which values of $n$ and $k$ does $V_{n}(k)$ extend to a representation of $S_{n+2}$ ?

By Whitehouse's result, for any $n>0$, the $k$-value $k=\left(\begin{array}{c}n-1 \\ 2\end{array}\right)$ leads to an extension as in Problem 10 . Also, since $V_{n}(0)$ is the trivial representation of $S_{n+1}$, one can take $k=0$ and $n$ arbitrary. On the other hand, if $k=1$ we have that $V_{n}(1)$ is the reflection representation of $S_{n+1}$. For $n>3$, this representation is not the restriction of any $S_{n+2}$-module.

\section{Acknowledgements}

B. Rhoades was partially supported by the NSF grant DMS-1068861. A. Berget was partially supported as VIGRE Fellow at UC Davis by NSF grant DMS-0636297.

A. Berget thanks Igor Pak for early discussions on determining the $S_{n+1}$-module structure of $V_{n}$.

\section{References}

[KW66] Alan G. Konheim and Benjamin Weiss. An occupancy discipline and applications. SIAM J. Applied Math., 14:1266-1274, 1966.

[PS04] Alexander Postnikov and Boris Shapiro. Trees, parking functions, syzygies, and deformations of monomial ideals. Trans. Amer. Math. Soc., 356(8):3109-3142 (electronic), 2004.

[Sag01] Bruce E. Sagan. The symmetric group, volume 203 of Graduate Texts in Mathematics. SpringerVerlag, New York, second edition, 2001. Representations, combinatorial algorithms, and symmetric functions.

[Sta82] Richard P. Stanley. Some aspects of groups acting on finite posets. J. Combin. Theory Ser. A, 32(2):132-161, 1982.

[Wac07] Michelle L. Wachs. Poset topology: tools and applications. In Geometric combinatorics, volume 13 of IAS/Park City Math. Ser., pages 497-615. Amer. Math. Soc., Providence, RI, 2007.

[Whi92] Neil White, editor. Matroid applications, volume 40 of Encyclopedia of Mathematics and its Applications. Cambridge University Press, Cambridge, 1992.

[Whi97] Sarah Whitehouse. The Eulerian representations of $\Sigma_{n}$ as restrictions of representations of $\Sigma_{n+1}$. J. Pure Appl. Algebra, 115(3):309-320, 1997. 